\title{
FORMALITY THEOREM FOR HOCHSCHILD (CO)CHAINS OF THE ALGEBRA OF ENDOMORPHISMS OF A VECTOR BUNDLE
}

\author{
VASILIY DolgusheV
}

\begin{abstract}
We prove the formality theorem for the differential graded Lie algebra module of Hochschild chains for the algebra of endomorphisms of a smooth vector bundle. We discuss a possible application of this result to a version of the algebraic index theorem for Poisson manifolds.
\end{abstract}

\section{Introduction}

The purpose of this note is to prove that the differential graded Lie algebra (DGLA) module of Hochschild chains for the algebra of endomorphisms of a smooth vector bundle over a manifold $M$ can be connected by a chain of quasi-isomorphisms to its cohomology.

For a trivial vector bundle one can easily prove the desired statement using the formality theorem [6], [7] for Hochschild chains of the algebra $\mathcal{O}_{M}$ of functions on $M$ and the (co)trace map [14] between the Hochschild complexes of the algebra $\mathcal{O}_{M}$ and the algebra $\operatorname{Mat}\left(\mathcal{O}_{M}\right)$ of finite size matrices with entries in $\mathcal{O}_{M}$.

However, for a non-trivial vector bundle $E$ both the trace and the cotrace maps between the Hochschild complexes of the algebra of functions and the algebra of endomorphisms are defined only locally. Thus, the question of formality of the DGLA module of Hochschild chains for the algebra of endomorphisms of a vector bundle requires some work.

In this note we propose Fedosov's resolution [7] of the sheaf of DGLA modules $\left(C^{\bullet}(\operatorname{End}(E)), C_{\bullet}(\operatorname{End}(E))\right)$ of Hochschild $(c o)$ chains for the sheaf $\operatorname{End}(E)$ of endomorphisms of a vector bundle $E$ and construct a quasi-isomorphism between this resolution and Fedosov's resolution of the sheaf of DGLA modules $\left(C^{\bullet}\left(\mathcal{O}_{M}\right), C_{\bullet}\left(\mathcal{O}_{M}\right)\right)$ for $\mathcal{O}_{M}$. Combining this construction with the result in [7] (see diagram (5.15) on page 86) we obtain a proof of the formality theorem for the sheaf of DGLA modules $\left(C^{\bullet}(\operatorname{End}(E)), C_{\bullet}(\operatorname{End}(E))\right)$.

Besides the obvious applications of this theorem to the questions of deformation quantization this result may lead to an interesting version of the algebraic index theorem for Poisson manifolds. We will devote a separate paper to this version of the algebraic index theorem. Here we will only outline a rough idea.

There are many versions of the algebraic index theorem for symplectic manifolds [2], [3], [5], [8] [15]. One of these versions [5] describes a natural map (see eq. (31) and theorem 4 in [5])

$$
c l: K_{0}(\mathbb{A}) \rightarrow H_{D R}^{\text {top }}(M)
$$

Received by the editors August 9, 2006. 
from the $K$-theory of the deformation quantization algebra $\mathbb{A}$ to the top degree De Rham cohomology of $M$. This map is obtained by composing the trace density map $[9]$

$$
\operatorname{trd}: \mathbb{A} /[\mathbb{A}, \mathbb{A}] \rightarrow H_{D R}^{\text {top }}(M)
$$

from the zeroth Hochschild homology $H H_{0}(\mathbb{A})=\mathbb{A} /[\mathbb{A}, \mathbb{A}]$ of $\mathbb{A}$ to the top degree De Rham cohomology of $M$ with the lowest component of the Chern character (see example 8.3.6 in [14])

$$
c h_{0,0}: K_{0}(\mathbb{A}) \rightarrow \mathbb{A} /[\mathbb{A}, \mathbb{A}]
$$

from the $K$-theory of $\mathbb{A}$ to the zeroth Hochschild homology of $\mathbb{A}$.

In the case of a general Poisson (not symplectic) manifold $M$ one cannot construct the trace density map (1). Instead we have the map

$$
\mu: \mathbb{A} /[\mathbb{A}, \mathbb{A}] \rightarrow H P_{0}(M)
$$

from zeroth Hochschild homology of $\mathbb{A}$ to the zeroth Poisson homology [4], [13] of $M$. This map is constructed with the help ${ }^{1}$ of the formality theorem for Hochschild chains [7], [16] of $\mathcal{O}_{M}$.

Composing (3) with (2) we get the map

$$
\text { ind }: K_{0}(\mathbb{A}) \rightarrow H P_{0}(M)
$$

from the $K$-theory of $\mathbb{A}$ to the zeroth Poisson homology of $M$.

Following the arguments of the proof of theorem 6.1.3 in [8] one can show that the image $\operatorname{ind}(Q)$ of a $K$-theory element $Q \in K_{0}(\mathbb{A})$ depends only on the principal part of $Q$. The desired algebraic index theorem should give an explicit form of this dependence and we expect that the formality theorem for Hochschild chains of $\operatorname{End}(E)$ will help us to solve this problem.

We would like to mention paper [11] by B. Keller. In this paper it is shown that the graded Lie algebra structure on Hochschild cohomology of an associative algebra is invariant with respect to the derived Morita equivalence. This result suggests a question of whether the Hochschild cochain complexes of the derived Morita equivalent associative algebras are quasi-isomorphic as DGLAs, and furthermore, as homotopy Gerstenhaber algebras.

A very short time after this paper was submitted, preprint [1] appeared on the web. In paper [1] the authors generalize the DGLA part of theorem 1 to the case of Azumaya algebras.

The paper is organized as follows. In the second section we fix notation and recall some results and constructions we are using here. In section 3 we formulate the main result of this paper (see theorem 1) and give a proof. In the concluding section we propose an interesting generalization of lemma 1 which is used in the proof of theorem 1. We suspect that this generalization may shed some light on an unknown index formula for the map (4).

\footnotetext{
${ }^{1}$ See corollary 2 on page 92 in [7].
} 


\section{Acknowledgments}

I would like to thank Volodya Rubtsov for stimulating discussions of this topic. I started this project when I was a Liftoff Fellow of Clay Mathematics Institute. I thank this Institute for the support. I am also partially supported by the Grant for Support of Scientific Schools NSh-8065.2006.2.

\section{Preliminaries}

In this section we fix notation and recall some results we are going to use in this note.

For an associative algebra $B$ we denote by $\operatorname{Mat}(B)$ the algebra of finite size matrices over $B . C \cdot(B)$ is the normalized Hochschild chain complex of $B$ with coefficients in $B$

$$
C_{\bullet}(B)=C_{\bullet}(B, B)
$$

and $C^{\bullet}(B)$ is the normalized Hochschild cochain complex of $B$ with coefficients in $B$ and with shifted grading

$$
C^{\bullet}(B)=C^{\bullet+1}(B, B) .
$$

The Hochschild coboundary operator is denoted by $\partial$ and the Hochschild boundary operator is denoted by $\mathfrak{b}$.

It is well known that the Hochschild cochain complex (6) carries the structure of a differential graded Lie algebra (DGLA). The corresponding Lie bracket (see eq. (3.2) on page 45 in [7]) was originally introduced by M. Gerstenhaber in [10]. We will denote this bracket by $[,]_{G}$.

The Hochschild chain complex (5) carries the structure of a differential graded Lie algebra module over the DGLA $C^{\bullet}(B)$. We will denote the action (see eq. (3.5) on page 46 in [7]) of cochains on chains by $R$.

The trace map $\operatorname{tr}[14]$ is the map from the Hochschild chain complex $C$. $(\operatorname{Mat}(B))$ of the algebra $\operatorname{Mat}(B)$ to the Hochschild chain complex $C_{\bullet}(B)$ of the algebra $B$. This map is defined by the formula

$$
\operatorname{tr}\left(M_{0} \otimes M_{1} \otimes \cdots \otimes M_{k}\right)=\sum_{i_{0}, \ldots, i_{k}}\left(M_{0}\right)_{i_{0} i_{1}} \otimes\left(M_{1}\right)_{i_{1} i_{2}} \otimes \cdots \otimes\left(M_{k}\right)_{i_{k} i_{0}},
$$

where $M_{0}, \ldots, M_{k}$ are matrices in $\operatorname{Mat}(B)$ and $\left(M_{a}\right)_{i j}$ are the corresponding entries.

Dually, the cotrace map [14]

$$
\operatorname{cotr}: C^{\bullet}(B) \rightarrow C^{\bullet}(\operatorname{Mat}(B))
$$

is defined by the formula

$$
\left(\operatorname{cotr}(P)\left(M_{0}, M_{1}, \ldots, M_{k}\right)\right)_{i j}=\sum_{i_{1}, \ldots, i_{k}} P\left(\left(M_{0}\right)_{i i_{1}},\left(M_{1}\right)_{i_{1} i_{2}}, \ldots,\left(M_{k}\right)_{i_{k} j}\right),
$$

where $P \in C^{k}(B)$ and $M_{0}, \ldots, M_{k}$ are, as above, matrices in $\operatorname{Mat}(B)$.

"DGLA" always means a differential graded Lie algebra. The arrow $\succ \longrightarrow$ denotes an $L_{\infty}$-morphism of sheaves of DGLAs, the arrow $\succ \succ \rightarrow$ denotes a morphism of sheaves 
of $L_{\infty}$-modules, and the notation

$$
\begin{gathered}
\mathcal{L} \\
\downarrow_{\bmod }
\end{gathered}
$$

$\mathcal{M}$

means that $\mathcal{M}$ is a sheaf of DGLA modules over the sheaf of DGLAs $\mathcal{L}$. The symbol o always stands for the composition of morphisms.

Throughout this note $M$ is a smooth real manifold of dimension $d . E$ is a smooth real vector bundle over $M$ and $\operatorname{End}(E)$ denotes the sheaf of endomorphisms of $E$. We denote by $\Gamma(M, \mathcal{G})$ the vector space of sections of the sheaf $\mathcal{G}$ and by $\Omega^{\bullet}(\mathcal{G})$ the sheaf of exterior forms with values in $\mathcal{G}$. We omit the symbol $\wedge$ referring to a local basis of exterior forms, as if one thought of $d x^{i}$ 's as anti-commuting variables.

$T_{\text {poly }}^{\bullet}$ is the sheaf of polyvector fields with shifted grading

$$
T_{\text {poly }}^{\bullet}=\wedge_{\mathcal{O}_{M}}^{\bullet+1} T M, \quad T_{\text {poly }}^{-1}=\mathcal{O}_{M}
$$

and $\mathcal{A}^{\bullet}$ is the sheaf of exterior forms.

$T_{\text {poly }}^{\bullet}$ is a sheaf of graded Lie algebras with respect the so-called Schouten-Nijenhuis bracket [, ] $]_{N}$ (see eq. (3.20) on page 50 in [7]) and $\mathcal{A}^{\bullet}$ is the sheaf of graded Lie algebra modules over $T_{\text {poly }}^{\bullet}$ with respect to Lie derivative (see eq. (3.21) on page 51 in [7]). We will regard $T_{\text {poly }}^{\bullet}$ (resp. $\mathcal{A}^{\bullet}$ ) as the sheaf of DGLAs (resp. the sheaf of DGLA modules) with vanishing differential.

We denote by $x^{i}$ local coordinates on $M$ and by $y^{i}$ fiber coordinates in the tangent bundle $T M$. Having these coordinates $y^{i}$ we can introduce another local basis of exterior forms $\left\{d y^{i}\right\}$. We will use both bases $\left\{d x^{i}\right\}$ and $\left\{d y^{i}\right\}$. In particular, the notation $\Omega^{\bullet}(\mathcal{G})$ is reserved for the sheaf of $d y$-exterior forms with values in the sheaf $\mathcal{G}$ while $\mathcal{A}^{\bullet}$ denotes the sheaf of $d x$-exterior forms.

$\mathcal{S} M$ is the formally completed symmetric algebra of the cotangent bundle $T^{*}(M)$. Sections of the sheaf $\mathcal{S} M$ can be viewed as formal power series in tangent coordinates $y^{i}$. We regard $\mathcal{S} M$ as the sheaf of algebras over $\mathcal{O}_{M}$. In particular, $C^{\bullet}(\mathcal{S} M)$ is the sheaf of normalized Hochschild cochains of $\mathcal{S} M$ over $\mathcal{O}_{M}$. Namely, the sections of $C^{k}(\mathcal{S} M)$ over an open subset $U \subset M$ are $\mathcal{O}_{M}$-linear polydifferential operators with respect to tangent coordinates $y^{i}$

$$
P: \Gamma(U, \mathcal{S} M)^{\otimes(k+1)} \rightarrow \Gamma(U, \mathcal{S} M)
$$

satisfying the normalization condition

$$
P(\ldots, f, \ldots)=0, \quad \forall f \in \mathcal{O}_{M}(U) .
$$

Similarly, $C_{\bullet}(\mathcal{S} M)$ is the sheaf of normalized Hochschild chains ${ }^{2}$ of $\mathcal{S} M$ over $\mathcal{O}_{M}$. As in [7] the tensor product in

$$
C_{k}(\mathcal{S} M)=\underbrace{\mathcal{S} M \hat{\otimes}_{\mathcal{O}_{M}}\left(\mathcal{S} M / \mathcal{O}_{M}\right) \hat{\otimes}_{\mathcal{O}_{M}} \ldots \hat{\otimes}_{\mathcal{O}_{M}}\left(\mathcal{S} M / \mathcal{O}_{M}\right)}_{k+1}
$$

is completed in the adic topology in fiber coordinates $y^{i}$ on the tangent bundle $T M$.

The cohomology of the complex of sheaves $C^{\bullet}(\mathcal{S} M)$ is the sheaf $\mathcal{T}_{\text {poly }}^{\bullet}$ of fiberwise polyvector fields (see page 60 in [7]). The cohomology of the complex of sheaves

\footnotetext{
${ }^{2}$ In [7] the sheaf $C^{\bullet}(\mathcal{S} M)$ is denoted by $\mathcal{D}_{\text {poly }}^{\bullet}$ and the sheaf $C \bullet(\mathcal{S} M)$ is denoted by $\mathcal{C}_{\bullet}^{\text {poly }}$.
} 
$C_{.}(\mathcal{S} M)$ is the sheaf $\mathcal{E}$ of fiberwise differential forms (see page 62 in [7]). These are $d x$-forms with values in $\mathcal{S} M$.

In [7] (see theorem 4 on page 68) it is shown that the sheaf of algebras $\Omega^{\bullet}(\mathcal{S} M)$ can be equipped with a differential of the following form

$$
D=\nabla-\delta+A,
$$

where

$$
\nabla=d y^{i} \frac{\partial}{\partial x^{i}}-d y^{i} \Gamma_{i j}^{k}(x) y^{j} \frac{\partial}{\partial y^{k}},
$$

is a torsion free connection with Christoffel symbols $\Gamma_{i j}^{k}(x)$,

$$
\delta=d y^{i} \frac{\partial}{\partial y^{i}},
$$

and

$$
A=\sum_{p=2}^{\infty} d y^{k} A_{k i_{1} \ldots i_{p}}^{j}(x) y^{i_{1}} \ldots y^{i_{p}} \frac{\partial}{\partial y^{j}} \in \Omega^{1}\left(M, \mathcal{T}_{\text {poly }}^{0}\right) .
$$

We refer to (9) as the Fedosov differential.

Notice that $\delta$ in (11) is also a differential on $\Omega^{\bullet}(\mathcal{S} M)$ and (9) can be viewed as deformation of $\delta$ via the connection $\nabla$.

Let us recall from [7] the following operator on ${ }^{3} \Omega^{\bullet}(\mathcal{S} M)$

$$
\delta^{-1}(a)=\left\{\begin{array}{cc}
y^{k} \frac{\vec{\partial}}{\partial\left(d y^{k}\right)} \int_{0}^{1} a(x, t y, t d y) \frac{d t}{t}, & \text { if } a \in \Omega^{>0}(U, \mathcal{S} M), \\
0, & \text { otherwise },
\end{array}\right.
$$

which is used to prove the acyclicity of $\delta$ and $D$ in positive dimension.

According to proposition 10 on page 64 in [7] the sheaves $\mathcal{T}_{\text {poly }}^{\bullet}, C^{\bullet}(\mathcal{S} M), \mathcal{E}^{\bullet}$, and $C_{\bullet}(\mathcal{S} M)$ are equipped with the canonical action of the sheaf of Lie algebras $\mathcal{T}_{\text {poly }}^{0}$ and this action is compatible with the corresponding (DG) algebraic structures. Using this action in chapter 4 of [7] we extend the Fedosov differential (9) to a differential on the sheaves $\Omega^{\bullet}\left(\mathcal{T}_{\text {poly }}^{\bullet}\right), \Omega^{\bullet}\left(\mathcal{E}^{\bullet}\right), \Omega^{\bullet}\left(C \bullet(\mathcal{S} M)\right.$ ), and $\Omega^{\bullet}\left(C^{\bullet}(\mathcal{S} M)\right.$ ) of DGLAs (resp. DGLA modules).

Using acyclicity of the Fedosov differential (9) in positive dimension one constructs in [7] embeddings of the sheaves of DGLA modules ${ }^{4}$

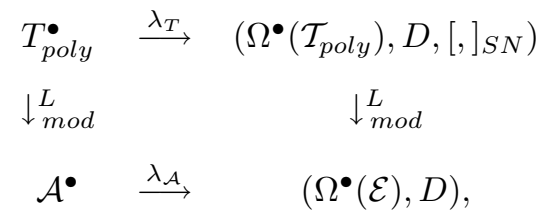

\footnotetext{
${ }^{3}$ The arrow over $\partial$ in (12) means that we use the left derivative with respect to the anti-commuting variable $d y^{k}$.

${ }^{4}$ See eq. (5.1) on page 81 in [7].
} 


$$
\begin{array}{ccc}
\left.\left(\Omega^{\bullet}(C \bullet \mathcal{S} M)\right), D+\partial,[,]_{G}\right) & \stackrel{\lambda_{D}}{\leftrightarrows} & C \cdot\left(\mathcal{O}_{M}\right) \\
\downarrow_{\text {mod }}^{R} & \downarrow_{\text {mod }}^{R} \\
\left(\Omega^{\bullet}(C \bullet(\mathcal{S} M)), D+\mathfrak{b}\right) & \stackrel{\lambda_{C}}{\longleftarrow} & C \bullet\left(\mathcal{O}_{M}\right),
\end{array}
$$

and shows that these are quasi-isomorphisms of the corresponding complexes of sheaves.

Furthermore, using Kontsevich's and Shoikhet's formality theorems for $\mathbb{R}^{d}[12]$, $[16]$ in [7] one constructs the following diagram

$$
\begin{array}{ccc}
\left(\Omega^{\bullet}\left(\mathcal{T}_{\text {poly }}\right), D,[,]_{S N}\right) & \succ \stackrel{\mathcal{K}}{\longrightarrow} & \left(\Omega^{\bullet}\left(C^{\bullet}(\mathcal{S} M)\right), D+\partial,[,]_{G}\right) \\
\downarrow_{\text {mod }}^{L} & & \downarrow_{\text {mod }}^{R} \\
\left(\Omega^{\bullet}(\mathcal{E}), D\right) & \stackrel{\mathcal{S}}{\prec \prec} & \left(\Omega^{\bullet}(C \bullet(\mathcal{S} M)), D+\mathfrak{b}\right)
\end{array}
$$

where $\mathcal{K}$ is an $L_{\infty}$ quasi-isomorphism of sheaves of DGLAs and $\mathcal{S}$ is a quasiisomorphism of sheaves of $L_{\infty}$-modules over the sheaf of DGLAs $\left(\Omega^{\bullet}\left(\mathcal{T}_{\text {poly }}\right), D,[,]_{S N}\right)$, and the $L_{\infty}$-module on $\Omega^{\bullet}(C \bullet(\mathcal{S} M))$ is obtained by composing the quasi-isomorphism $\mathcal{K}$ with the DGLA modules structure $R$ (see eq. (3.5) on p. 46 in [7] for the definition of $R$ ).

Diagrams (13), (14) and (15) show that the sheaf $C \cdot\left(\mathcal{O}_{M}\right)$ of DGLA modules of Hochschild chains of $\mathcal{O}_{M}$ is quasi-isomorphic to the sheaf of graded Lie algebra modules $\mathcal{A}^{\bullet}$ of its cohomology.

Remark 1. As in [7] we use adapted versions of Hochschild (co)chains for the sheaves $\mathcal{O}_{M}$ and $\operatorname{End}(E)$ of functions and of endomorphisms of a vector bundle $E$, respectively. Thus, $C^{\bullet}\left(\mathcal{O}_{M}\right)$ is a sheaf of polydifferential operators (see page 48 in [7]) satisfying the corresponding normalization condition. $C^{\bullet}(\operatorname{End}(E))$ is the sheaf of (normalized) polydifferential operators acting on $\operatorname{End}(E)$ with coefficients in $\operatorname{End}(E)$. Furthermore, $C \cdot\left(\mathcal{O}_{M}\right)$ is the sheaf of (normalized) polyjets

$$
C_{k}\left(\mathcal{O}_{M}\right)=H_{o m} \mathcal{O}_{M}\left(C^{k-1}\left(\mathcal{O}_{M}\right), \mathcal{O}_{M}\right),
$$

and

$$
C_{k}(\operatorname{End}(E))=H o m_{\operatorname{End}(E)}\left(C^{k-1}(\operatorname{End}(E)), \operatorname{End}(E)\right) .
$$

We have to warn the reader that the space of global sections of the sheaf $C^{\bullet}\left(\mathcal{O}_{M}\right)$ (resp. $\left.C^{\bullet}(\operatorname{End}(E))\right)$ is not isomorphic to the space of Hochschild cochains of the algebra $\mathcal{O}_{M}(M)$ of functions (resp. the algebra $\Gamma(M, \operatorname{End}(E)$ ) of endomorphisms of $E)$. Similar expectation is wrong for Hochschild chains. Instead we have the following inclusions:

$$
\begin{array}{lll}
\Gamma\left(M, C^{\bullet}\left(\mathcal{O}_{M}\right)\right) \subset C^{\bullet}\left(\mathcal{O}_{M}(M)\right), & \Gamma\left(M, C^{\bullet}(\operatorname{End}(E))\right) \subset C^{\bullet}(\Gamma(M, \operatorname{End}(E))), \\
C_{\bullet}\left(\mathcal{O}_{M}(M)\right) \subset \Gamma\left(M, C_{\bullet}\left(\mathcal{O}_{M}\right)\right), & C_{\bullet}(\Gamma(M, \operatorname{End}(E))) \subset \Gamma(M, C \bullet(\operatorname{End}(E))) .
\end{array}
$$

Remark 2. Unlike in [7] we use only normalized Hochschild (co)chains. It is not hard to check that the results we need from [7], [12], and [16] also hold when this normalization condition is imposed. 


\section{The formality theorem}

Let $E$ be a smooth real vector bundle over the smooth real manifold $M$ and let $\operatorname{End}(E)$ denote the sheaf of endomorphisms of $E$. We regard $\operatorname{End}(E)$ as a sheaf of algebras over $\mathbb{R}$.

Here is the main result of this note:

Theorem 1. The sheaf of DGLA modules $C \cdot(\operatorname{End}(E))$ over the sheaf of DGLAs $C^{\bullet}(\operatorname{End}(E))$ is formal.

Proof. Let us introduce the following auxiliary sheaf of algebras

$$
\mathrm{ES}=\operatorname{End}(E) \otimes_{\mathcal{O}_{M}} \mathcal{S} M
$$

Regarding $\mathrm{E} \mathcal{S}$ as a sheaf of algebras over $\mathcal{O}_{M}$ we also consider the following adapted versions of (normalized) Hochschild (co)chains. Thus, $C^{k}(\mathrm{ES})$ is the sheaf whose sections over an open subset $U \subset M$ are $\mathcal{O}_{M}$-polylinear maps

$$
P: \Gamma(U, \mathrm{E} \mathcal{S})^{\otimes k+1} \rightarrow \Gamma(U, \mathrm{E} \mathcal{S}),
$$

which are differential in fiber coordinates $y^{i}$ and satisfy the normalization condition:

$$
P(\ldots, f, \ldots)=0, \quad \forall \quad f \in \mathcal{O}_{M}(U) .
$$

Similarly, $C_{k}(\mathrm{ES})$ is the sheaf of normalized Hochschild chains of ES over $\mathcal{O}_{M}$ for which the tensor product is completed in the adic topology in fiber coordinates $y^{i}$ on the tangent bundle $T M$.

It is clear that the differentials $\partial, \mathfrak{b}$ as well as the operations [, $]_{G}$ and $R$ are well defined on the sheaves $C^{\bullet}(\mathrm{ES}), C_{\bullet}(\mathrm{ES})$. Thus, we regard $C_{\bullet}(\mathrm{ES})$ as the sheaf of DGLA modules over the sheaf of DGLAs $C^{\bullet}(\mathrm{ES})$.

It is not hard to show that one can extend the Fedosov differential (9) on $\mathcal{S} M$ to a differential on $\mathrm{ES}$ in the framework of the following ansatz:

$$
\widetilde{D}=D+\left[\gamma^{E},\right], \quad \gamma^{E}=\Gamma^{E}+\widetilde{\gamma}^{E},
$$

where $\Gamma^{E}$ is a connection form of $E$ and $\widetilde{\gamma}^{E}$ is a section of $\Omega^{1}(\mathrm{ES})$.

More precisely, we first extend the operator $\delta^{-1}(12)$ to $\Omega^{\bullet}(\mathrm{ES})$ and then define $\gamma^{E}$ as a result of iterating the following equation (in degrees in $y$ )

$$
\gamma^{E}=\Gamma^{E}+\delta^{-1}\left(\nabla \gamma^{E}+A\left(\gamma^{E}\right)+\frac{1}{2}\left[\gamma^{E}, \gamma^{E}\right]\right) .
$$

Then $\gamma^{E}$ satisfies the identity

$$
D \gamma^{E}+\frac{1}{2}\left[\gamma^{E}, \gamma^{E}\right]=0
$$

which immediately implies that $\widetilde{D}^{2}=0$.

The differential $\widetilde{D}(17)$ naturally extends to the sheaf of DGLAs $\Omega^{\bullet}\left(C^{\bullet}(\mathrm{ES})\right)$ and to the sheaf of DGLA modules $\Omega^{\bullet}(C \bullet$ ES $\left.)\right)$. Namely, on $\Omega^{\bullet}\left(C^{\bullet}(\mathrm{ES})\right) \widetilde{D}$ is defined by the formula

$$
\widetilde{D}=D+\left[\partial \gamma^{E},\right]_{G},
$$

and on $\Omega^{\bullet}\left(C_{\bullet}(\mathrm{ES})\right) \widetilde{D}$ is defined by

$$
\widetilde{D}=D+R_{\partial \gamma^{E}}
$$


where $\gamma^{E}$ is viewed locally as a section of the sheaf $\Omega^{1}\left(C^{-1}(\mathrm{ES})\right)$ and $\partial$ denotes the Hochschild coboundary operator.

Since the sheaf of DGLA modules

$$
\begin{gathered}
\left(\Omega^{\bullet}\left(C^{\bullet}(\mathcal{S} M)\right), D+\partial,[,]_{G}\right) \\
\downarrow_{\bmod } \\
\left(\Omega^{\bullet}\left(C_{\bullet}(\mathcal{S} M)\right), D+\mathfrak{b}\right)
\end{gathered}
$$

is connected by a chain of quasi-isomorphisms to its cohomology $(13),(14),(15)$ it suffices to show that $(22)$ is quasi-isomorphic to the sheaf of DGLA modules $C \bullet(\operatorname{End}(E))$ over the sheaf of DGLAs $C^{\bullet}(\operatorname{End}(E))$. It is the sheaf of DGLA modules

$$
\left(\Omega^{\bullet}\left(C^{\bullet}(\mathrm{ES})\right), \widetilde{D}+\partial,[,]_{G}\right)
$$

$$
\begin{gathered}
\downarrow_{\bmod } \\
\left(\Omega^{\bullet}\left(C_{\bullet}(\mathrm{ES})\right), \widetilde{D}+\mathfrak{b}\right)
\end{gathered}
$$

which allows us to do it.

Indeed, generalizing the construction of the maps $\lambda_{D}$ and $\lambda_{C}$ in (14) we get the following embeddings of the sheaves of DGLA modules

$$
\begin{aligned}
& \left(\Omega^{\bullet}\left(C^{\bullet}(\mathrm{ES})\right), \widetilde{D}+\partial,[,]_{G}\right) \quad \stackrel{\lambda_{D}^{E}}{\longleftarrow} \quad C^{\bullet}(\operatorname{End}(E)) \\
& \downarrow_{\bmod }^{R} \quad \downarrow_{\bmod }^{R} \\
& \left(\Omega^{\bullet}\left(C_{\bullet}(\mathrm{ES})\right), \widetilde{D}+\mathfrak{b}\right) \quad \stackrel{\lambda_{C}^{E}}{\longleftarrow} \quad C_{\bullet}(\operatorname{End}(E)) .
\end{aligned}
$$

Similarly to propositions 7, 13, 15 in [7] one can easily show that $\lambda_{D}^{E}$ and $\lambda_{C}^{E}$ are quasi-isomorphisms of the corresponding complexes of sheaves.

Thus it remains to connect the sheaf of DGLA modules (23) to (22) by a quasiisomorphism. To do this we need the following auxiliary statement which is proved in a more general form in the concluding section

Lemma 1. Let $a, b, c, d$ be elements of a graded associative algebra with the degrees

$$
\operatorname{deg} a=0, \quad \operatorname{deg} b=\operatorname{deg} c=\operatorname{deg} d=1
$$

and let a be nilpotent. If these elements satisfy the following relations

$$
\begin{gathered}
{[d, a]=b-\frac{c}{2}, \quad[b, a]=c,} \\
{[c, a]=0,}
\end{gathered}
$$

then

$$
d \exp (a)=\exp (a)(d+b)
$$

Let us pick a trivialization of $E$ over a neighborhood $V$ of a point $p \in M$ and notice that on $V$ the initial Fedosov differential (9) on the sheaves $\Omega^{\bullet}\left(C^{\bullet}(\mathrm{ES})\right), \Omega^{\bullet}\left(C_{\bullet}(\mathrm{ES})\right)$ is well defined and the connection form $\gamma^{E}$ (18) can be viewed as a section of the sheaf $\left.\Omega^{1}(\mathrm{ES})\right|_{V}$. 
Furthermore, over $V$ the trace and cotrace maps give the following commutative diagram of the quasi-isomorphisms of the sheaves of DGLAs and their modules

$$
\begin{array}{ccc}
\left(\Omega^{\bullet}\left(C^{\bullet}(\mathcal{S} M)\right), D+\partial,[,]_{G}\right) & \stackrel{\operatorname{cotr}}{\longrightarrow} & \left(\Omega^{\bullet}\left(C^{\bullet}(\mathrm{ES})\right), D+\partial,[,]_{G}\right) \\
\downarrow_{\text {mod }} & & \downarrow_{\text {mod }} \\
\left(\Omega^{\bullet}(C \bullet(\mathcal{S} M)), D+\mathfrak{b}\right) & \stackrel{\operatorname{tr}}{\leftarrow} & \left(\Omega^{\bullet}(C \bullet(\mathrm{ES})), D+\mathfrak{b}\right) .
\end{array}
$$

Now we notice that (19) implies the identities

$$
\begin{gathered}
{\left[(D+\mathfrak{b}), R_{\gamma^{E}}\right]=R_{\partial \gamma^{E}}-\frac{1}{2} R_{\left[\partial \gamma^{E}, \gamma^{E}\right]_{G}},} \\
{\left[(D+\partial),\left[\gamma^{E},\right]_{G}\right]=\left[\partial \gamma^{E},\right]_{G}-\frac{1}{2}\left[\left[\partial \gamma^{E}, \gamma^{E}\right]_{G},\right]_{G},} \\
{\left[\left[\partial \gamma^{E}, \gamma^{E}\right]_{G}, \gamma^{E}\right]_{G}=0,}
\end{gathered}
$$

which allow us to apply lemma 1 to the algebras of operations on the sheaves $\Omega^{\bullet}\left(C^{\bullet}(\mathrm{ES})\right.$ and $\Omega^{\bullet}\left(C_{\bullet}(\mathrm{ES})\right.$.

Indeed, setting

$$
a=\left[\gamma^{E},\right]_{G}, \quad b=\left[\partial \gamma^{E},\right]_{G}, \quad c=\left[\left[\partial \gamma^{E}, \gamma^{E}\right]_{G},\right]_{G}, \quad d=D+\partial
$$

and using (29) and (30) we get that the map of complexes of sheaves (over $V$ )

$$
\exp \left(-\left[\gamma^{E},\right]_{G}\right): \Omega^{\bullet}\left(C^{\bullet}(\mathrm{ES}), D+\partial\right) \rightarrow\left(\Omega^{\bullet}\left(C^{\bullet}(\mathrm{E} \mathcal{S}), \widetilde{D}+\partial\right)\right.
$$

is compatible with the corresponding differentials.

Similarly, setting

$$
a=R_{\gamma^{E}}, \quad b=R_{\partial \gamma^{E}}, \quad c=R_{\left[\partial \gamma^{E}, \gamma^{E}\right]_{G}}, \quad d=D+\mathfrak{b}
$$

and using (28) and (30) we get that the map of complexes of sheaves (over $V$ )

$$
\exp \left(R_{\gamma^{E}}\right):\left(\Omega ^ { \bullet } ( C _ { \bullet } ( \mathrm { ES } ) , \widetilde { D } + \mathfrak { b } ) \rightarrow \left(\Omega^{\bullet}(C \bullet(\mathrm{ES}), D+\mathfrak{b})\right.\right.
$$

is also compatible with the differentials.

Combining these results with (27) we get the following commutative diagram of maps of sheaves of DGLAs and their modules (over the neighborhood $V$ )

$$
\begin{aligned}
& \left(\Omega^{\bullet}\left(C^{\bullet}(\mathcal{S} M)\right), D+\partial,[,]_{G}\right) \stackrel{\operatorname{cotr}^{t w}}{\longrightarrow}\left(\Omega^{\bullet}\left(C^{\bullet}(\mathrm{ES})\right), \widetilde{D}+\partial,[,]_{G}\right)
\end{aligned}
$$

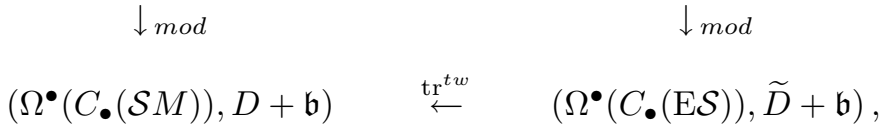

where

$$
\operatorname{cotr}^{t w}=\exp \left(-\left[\gamma^{E},\right]_{G}\right) \circ \operatorname{cotr}, \quad \operatorname{tr}^{t w}=\operatorname{tr} \circ \exp \left(R_{\gamma^{E}}\right) .
$$

Under changing the trivialization $\gamma^{E}$ gets replaced by $\gamma^{E}+\Delta$, where $\Delta$ is a one-form in $\Omega^{1}(\mathrm{ES})$ which does not involve the tangent coordinates $y^{i}$. Thus the maps (34) do not depend on the choice of trivialization because we deal normalized Hochschild (co)chains. 
Using the descending filtration associated to the exterior degree on the sheaves in (33) one can easily show that the maps $\operatorname{tr}^{t w}$ and $\operatorname{cotr}^{t w}$ are quasi-isomorphisms of complexes of sheaves.

The theorem is proved.

\section{Concluding remarks}

In this section we show that lemma 1 admits an interesting generalization that involves the function

$$
f(x)=\frac{x}{e^{x}-1}
$$

from the definition of the Todd class.

This generalization can be formulated as

Proposition 1. Let $a, b, d$ be elements of a graded associative algebra with the degrees

$$
\operatorname{deg} a=0, \quad \operatorname{deg} b=\operatorname{deg} d=1 .
$$

Let a be nilpotent and let $\alpha_{1}, \alpha_{2}, \ldots, \alpha_{k}, \ldots$ be coefficients of the Taylor power series for the function

$$
\frac{x}{e^{x}-1}=1+\alpha_{1} x+\alpha_{2} x^{2}+\ldots
$$

Then the relation

$$
\exp (a) d=(d+b) \exp (a)
$$

holds if and only if

$$
[a, d]=b+\sum_{k=1}^{\infty} \alpha_{k}\left(a d_{a}\right)^{k} b
$$

Proof. Since $a$ is a nilpotent element all infinite power series in $a$ or in $a d_{a}$ are well defined.

Equation (37) can be rewritten as

$$
[a, d]=f\left(a d_{a}\right) b,
$$

where $f=f(x)$ is given in (35) and the operator $f\left(a d_{a}\right)$ is defined via the Taylor expansion of $f$ around the point $x=0$

$$
f\left(a d_{a}\right)=I d+\sum_{k=1}^{\infty} \alpha_{k}\left(a d_{a}\right)^{k} .
$$

It is clear that (38) holds if and only if

$$
b=g\left(a d_{a}\right)[a, d],
$$

where $g(x)=\frac{e^{x}-1}{x}$ and $g\left(a d_{a}\right)$ is also defined via the corresponding Taylor expansion.

The latter equation is equivalent to

$$
b=\exp \left(a d_{a}\right) d-d
$$

and this is exactly what we need to prove. 
Lemma 1 together with its generalization come to us as surprise. We hope that the relation with the formula for the Todd class can be helpful in deriving a version of the algebraic index theorem for the map (4).

\section{References}

[1] P. Bressler, A. Gorokhovsky, R. Nest, and B. Tsygan, Deformations of Azumaya algebras, math.QA/0609575.

[2] P. Bressler, R. Nest, and B. Tsygan, Riemann-Roch theorems via deformation quantization. I, Adv. Math. 167 (2002), no. 1, 1-25.

[3] _ Riemann-Roch theorems via deformation quantization. II, Adv. Math. 167 (2002), no. 1, 26-73.

[4] J.-L. Brylinski, A differential complex for Poisson manifolds, J. Diff. Geom. 28 (1988), no. 1, $93-114$.

[5] P. Chen and V.A. Dolgushev, A Simple Algebraic Proof of the Algebraic Index Theorem, Math. Res. Lett. 12 (2005), no. 5-6, 655 - 671.

[6] V.A. Dolgushev, A Formality theorem for Hochschild chains, Adv. Math. 200 (2006), no. 1, 51-101.

[7] _ A Proof of Tsygan's formality conjecture for an arbitrary smooth manifold, PhD thesis, MIT; math.QA/0504420.

[8] B.V. Fedosov, Deformation quantization and index theory, Mathematical Topics, 9. Akademie Verlag, Berlin, 1996

[9] B. Feigin, G. Felder, and B. Shoikhet, Hochschild cohomology of the Weyl algebra and trace in deformation quantization, Duke Math. J. 127 (2005), no. 3, 487 - 517.

[10] M. Gerstenhaber, The cohomology structure of an associative ring, Ann. of Math. (2) $\mathbf{7 8}$ (1963) 267-288.

[11] B. Keller, Hochschild cohomology and derived Picard groups, J. Pure Appl. Algebra 190 (2004), no. 1-3, 177-196.

[12] M. Kontsevich, Deformation quantization of Poisson manifolds, Lett. Math. Phys. 66 (2003), no. $3,157-216$

[13] J.L. Koszul, Crochet de Schouten-Nijenhuis et cohomologie, Astérisque (1985) Numero Hors Serie, $257-271$

[14] J.- L. Loday, Cyclic Homology, Grundlehren der mathematischen Wissenschaften, 301. Springer-Verlag, Berlin, 1992.

[15] R. Nest and B Tsygan, Algebraic index theorem, Commun. Math. Phys. 172 (1995), no. 2, 223-262.

[16] B. Shoikhet, A proof of the Tsygan formality conjecture for chains, Adv. Math. 179 (2003), no. $1,7-37$.

Department of Mathematics, Northwestern University, Evanston, IL 60208

E-mail address: vald@math.northwestern.edu 